\title{
Development and Open Trial of a Depression Preventive Intervention for Adolescents With Attention-Deficit/Hyperactivity Disorder
}

\author{
Michael C. Meinzer, \\ University of Maryland \\ Chelsey M. Hartley, \\ Florida International University \\ Katherine Hoogesteyn, \\ Universiteit Maastricht \\ Jeremy W. Pettit \\ Florida International University
}

\begin{abstract}
Adolescents with attention-deficit/hyperactivity disorder (ADHD) are at elevated risk for experiencing unipolar depressive symptoms and disorders. The current study describes the development of a behaviorally oriented depression preventive intervention tailored for adolescents with ADHD targeting variables empirically shown to mediate ADHD and depression (i.e., reward responsivity, emotion regulation, and family support). Eight adolescents with a history of ADHD and currently elevated depressive symptoms and their parents participated in an open trial of the Behaviorally Enhancing Adolescents' Mood (BEAM) program. Adolescents and their parents reported high satisfaction with BEAM. Staff reported BEAM was easy to implement with high adherence. Following BEAM, there were significant reductions in parent-report of adolescents' depressive symptoms and emotion regulation at posttreatment and the 6-week follow-up and adolescent-report of reward responsivity at posttreatment. Case vignettes are also provided to illustrate implementation of the BEAM program. In spite of the small sample, lack of a control group, and some discrepancies across informants, results overall support the feasibility and acceptability of the BEAM program, and suggest it has promise in reducing depressive symptoms in adolescents with ADHD.
\end{abstract}

\section{Keywords}

ADHD; depression; adolescence; comorbidity; preventive intervention

THOUGH once considered a disorder of childhood, research has established that attentiondeficit/ hyperactivity disorder (ADHD) persists into or even worsens in adolescence (Molina

Address correspondence to Michael C. Meinzer, Ph.D., Department of Psychology, University of Maryland, College Park, MD 20742; mmeinzer@umd.edu. 
et al., 2009; Wolraich et al., 2005). ADHD is associated with high levels of depressive symptoms and high rates of unipolar depressive disorders in childhood and adolescence (for a meta-analysis, see Meinzer, Pettit, \& Viswesvaran, 2014; for exceptions, see Claude \& Firestone, 1995; Mannuzza et al., 1991; Mannuzza, Klein, Bessler, Malloy, \& LaPadula, 1998). Rates of depression are 5.5 times higher in youth with versus without ADHD (Angold, Costello, \& Erkanli, 1999), and 12\% to 50\% of youth with ADHD will meet criteria for major depressive disorder (MDD; Biederman et al., 2008; Biederman, Newcorn, \& Sprich, 1991; Jensen, Burke, \& Garfinkel, 1988). The co-occurrence of ADHD and depression represents a pressing concern given the higher impairment seen in adolescents who experience both disorders as compared to either disorder in isolation (Biederman et al., 1991). Given the elevated risk of depression seen in adolescents with ADHD as well as the associated impairment, there is a need for programs to prevent and reduce depressive symptoms among adolescents with ADHD. The present study represents a step toward addressing that need by developing a depression preventive intervention (Behaviorally Enhancing Adolescents' Mood; BEAM) for adolescents with ADHD and evaluating it in an open trial (i.e., a pilot study without the use of a control group).

\section{The BEAM Program: Rationale and Description}

Development of the BEAM program was informed by empirical research on mediators of the association between ADHD and depression: reward responsivity, emotion regulation, and family support. Reward responsivity, or differences in reaction to pleasurable stimuli and reward (Bogdan \& Pizzagalli, 2009), has been linked to both ADHD and depression in neuroscience and genetic frameworks (e.g., Durston, 2003; Kato, 2007; Scheres et al., 2007; Wood \& Neale, 2010) and has been shown to account for the covariation between ADHD and depressive symptoms (Meinzer et al., 2012). Deficits in emotion regulation (i.e., the awareness, understanding, acceptance, and appropriate modulation of emotions; Gratz \& Roemer, 2004) also have been linked to ADHD and depression, with large effect sizes between ADHD and emotion regulation (e.g., Walcott \& Landau, 2004) and medium effect sizes between depression and emotion regulation (e.g., Durbin \& Shafir, 2008). Youth with ADHD use less adaptive emotion regulation strategies (Melnick \& Hinshaw, 2000) and have more difficulty recognizing and characterizing negative emotions compared to youth without ADHD (Norvilitis, Casey, Brooklier, \& Bonello, 2000). Deficits in emotion regulation have been found to concurrently and prospectively predict depression in youth (Durbin \& Shafir, 2008; Feng et al., 2009; Kuyken, Watkins, Holden, \& Cook, 2006) and to mediate the association between ADHD and depression in youth (Anastopoulos et al., 2011; Seymour et al., 2012; Seymour et al., 2014). Finally, poor family support has been linked to ADHD and depression. The negative-reactive response pattern theory (Johnston, 1996) asserts that youth with ADHD display higher rates of disruptive behavior within the family context compared to their non-ADHD peers, including less compliance to parent's directions, more hyperactive behavior, and less on-task behavior (Cunningham \& Barkley, 1979; Johnston, 1996). Youths' disruptive behavior elicits commanding and disapproving behavior from parents (Wells et al., 2006), which further contributes to the youth's behavior difficulties (Cunningham \& Barkley, 1979), exacerbating the negative parent-child relationship and leading to low family 
support. Family support has also been found to mediate the association between ADHD and depressive symptoms (e.g., Humphreys et al., 2013; Meinzer, Hill, et al., 2014).

BEAM was designed to explicitly target reward responsivity, emotion regulation, and family support and was tailored to meet the developmental and cognitive levels of adolescents with ADHD. Many existing depression preventive interventions heavily emphasize cognitive strategies such as cognitive restructuring and thought replacement (e.g., Stice, Shaw, Bohon, Marti, \& Rohde, 2009). Complex cognitive strategies typically have not been effective among youth with ADHD (Smith, Barkley, \& Shapiro, 2006). Thus, BEAM was developed with an eye toward minimizing cognitive strategies and instead emphasized behavioral strategies (e.g., Dimidjian, Barrera, Martell, Munoz, \& Lewinsohn, 2011; Hopko, Lejuez, Ruggiero, \& Eifert, 2003) to maximize skill building through active exercises and behavioral learning principles that require minimal didactic instruction.

Behavioral models of depression posit that a combination of social skills deficits and minimal availability of and restricted range of positively reinforcing events lead to low rates of response contingent positive reinforcement, which in turn lead to dysphoria and somatic symptoms of depression (Lewinsohn, 1974). Stemming from this perspective, pleasant activity scheduling and mood monitoring are intervention techniques that can be used to help adolescents identify connections between engagement in activities and affective states, thus leading to an increase in response contingent positive reinforcement and a decrease in symptoms of depression (Dimidjian et al., 2011; Mazzuchelli, Kane, \& Rees, 2009). In accordance with these behavioral models, pleasant activity scheduling was used to increase adolescents' access to positively reinforcing and pleasant events (Hopko et al., 2003). This approach has demonstrated similar efficacy to CBT and interpersonal skills training approaches (Zeiss, Lewinsohn, \& Munoz, 1979) and has been applied to adolescent patients (McCauley et al., 2011; Ritschel, Ramirez, Jones, \& Craighead, 2011).

BEAM was tailored for adolescents with ADHD who experience elevated, yet subthreshold, levels of depressive symptoms. Over half of adolescents with ADHD do not develop MDD by age 30 (Meinzer et al., 2013). Providing a depression prevention program to all adolescents with ADHD would therefore represent an inefficient use of limited mental health resources. A more efficient use of limited resources would be to identify adolescents who already experience subthreshold depressive symptoms and thus are at heightened risk of developing MDD. The presence of subthreshold depressive symptoms also ensured that strategies in BEAM could be taught, applied, and practiced in the context of current depressive symptoms. BEAM thus represents a hybrid selective-indicated prevention program, in that participants are selected on the basis of an established risk factor (ADHD) for depression as well as the presence of subthreshold depressive symptoms.

The present study reports on the development of BEAM and preliminary findings from an open trial. The primary aims of the open trial were to evaluate the feasibility of BEAM and participating families' satisfaction with BEAM. Examples of intervention techniques and clinical vignettes to illustrate implementation of the BEAM program are also included. Secondary aims were to preliminarily examine the influence of BEAM on adolescents' levels of depressive symptoms, reward responsivity, emotion regulation, and family support.

Cogn Behav Pract. Author manuscript; available in PMC 2019 November 29. 


\section{Method}

\section{Participants}

Eight adolescents and their parents were enrolled in the open trial of BEAM. Adolescents ranged in age from 12 to 16 years $(M=13.00, S D=1.31)$ and were primarily male (75\%) and Hispanic (75\%). Demographic information for each participant is presented in Table 1.

For study inclusion, adolescents were required to (a) meet criteria for a lifetime DSM-IV diagnosis of ADHD, (b) display currently elevated depressive symptoms as indicated by either parent-rating or adolescent self-rating (i.e., a $T$-score $>65$ on the Children's Depression Inventory- $2^{\text {nd }}$ edition; Kovacs, 2011), and (c) have previously received psychosocial/behavioral treatment for ADHD. With regards to Criterion B, given that there may be differences in informant perspectives that cause parent and child self-ratings to be weakly to moderately correlated (Achenbach, Dumenci, \& Rescorla, 2003) and that the focus of the proposed study was preventive intervention, a sensitive approach was used such that adolescents were eligible for inclusion if either the parent or adolescent reported elevated adolescent depressive symptoms. Criterion $\mathrm{C}$ was made to ensure parents would have a basic understanding of behavioral treatment principles. As an adjunctive treatment program, it was necessary that parents had a basic framework for the behavioral treatment of ADHD. Adolescents were not required to be free of ADHD symptoms and impairment but rather to have participated in parent training or a behavioral treatment program for ADHD. The treatment programs parents had previously participated in ranged from weekly 3-hour sessions with child treatment components to traditional weekly appointments for 1 hour. These programs emphasized establishing rewards for desired behaviors (e.g., following directions, completing homework) and negative consequences for ADHD-related impairment. Thus, BEAM was developed as an adjunctive therapy to behavioral treatments for ADHD.

Exclusion criteria were: (a) a lifetime history of MDD, dysthymia, bipolar disorder, pervasive developmental disorder, schizophrenia and/or any other psychotic or organic mental disorders, (b) inability to understand or communicate in English, and (c) IQ below 80 (estimated using the matrix reasoning and vocabulary subscales of the Wechsler Abbreviated Scale for Intelligence).

\section{Design and Methods}

Recruitment, Phone Screening, and Pretreatment Assessment-Participants were recruited through a database of families that had previously received services for ADHD at the investigators' university-affiliated clinic. Currently elevated adolescent depressive symptoms on the CDI-2 (i.e., a Tscore > 65; Kovacs, 2011) were endorsed by either a parent, child, or both for all 8 families. These 8 families completed a pretreatment assessment where parents and adolescents completed psychosocial rating scales, behavior observation tasks, and a diagnostic interview to establish inclusion/exclusion criteria. All 8 families met inclusion/exclusion criteria. Families were also asked to maintain ADHD medication status at a stable dose during the course of the study and to inform the research team if they desired to alter or begin medication during the course of the study. Medication 
requirements were made to ensure symptom changes and behavior changes in the open trial were not due to other psychosocial interventions or changes in pharmacological interventions. Five adolescents $(62.5 \%)$ had taken medication for ADHD in the past and 3 (37.5\%) were currently taking medication for ADHD. No families indicated any changes to their medication regimen during the course of the study.

All parents reported that they previously participated in behavioral treatment of ADHD during their initial assessment, as per the study's inclusion criteria. We did not assess parents' level of knowledge or skills with behavioral treatment programs.

Families were offered the four-session BEAM intervention at no cost. Following the BEAM program, families completed assessments at posttreatment, 6-week follow-up, and 12-week follow-up. Families were not compensated financially for their participation.

\section{The BEAM Program}

BEAM is a four-session, group-based preventive intervention that included both an adolescent and a parent component. The BEAM intervention tailors existing evidence-based strategies such as pleasant events scheduling and problem solving and implements them in a format designed to meet the needs of adolescents with ADHD. Parents and adolescents met for 2.5 hours on Saturday mornings for 4 consecutive weeks. Parents and adolescents met in separate groups for the first 2 hours, then met in a combined group for 30 minutes to work on problem-solving skills through the use of activities and group discussions. Parental involvement in BEAM was deemed important to (a) facilitate adolescents' implementation and practice of BEAM strategies at home and (b) target family support by addressing problem-solving deficits and interpersonal conflict from both adolescents' and parents' perspectives. Though parents and adolescents were separated for the majority of session time, the material covered in each session coincided so that parents and adolescents could review and practice material together during the week. An overview of material covered in each adolescent and parent session is presented in Table 2. Parents and adolescents each received workbooks that they took home with them following each session.

Program Staff-Two doctoral students in clinical psychology served as the primary treatment providers, one of whom was the treatment developer. Both providers had previous training in and experience implementing behavioral intervention for ADHD. Prior to beginning treatment, providers read through and discussed manual material. Treatment providers met on a weekly basis to review content to be covered in the upcoming session as well as issues that arose during the previous session. Supervision was provided by a licensed clinical psychologist. BEAM utilized a relatively simple delivery format that did not require complex skills from the patient or the provider. BEAM was designed to be easily implemented in the community by mental health professionals with varying degrees of expertise.

Adolescent Component-The adolescent component of BEAM consisted of modules on reward responsivity, emotion regulation, and family support to target the empirically identified mediators of the association between ADHD and depression. These modules were interwoven into each session. 
Reward responsivity.: Stemming from Lewinsohn's behavioral model of depression (1974), BEAM targeted reward responsivity by fostering participation in a higher frequency of positively reinforcing events and having participants explicitly draw linkages between positive activities and daily mood ratings. The goal was to increase anticipatory pleasure (i.e., how much I think I will like the activity) and consummatory pleasure (i.e., how much I like the activity in the moment). Thus, pleasant activity scheduling was implemented. Reward responsivity was selected and described as a target given that it has been empirically identified as a mediator. Pleasant activity scheduling (and behavior activation in terms of scheduling "productive activities") was selected as an intervention strategy that would lead to changes in the targeted variable, reward responsivity.

Also, the preventive intervention used in-vivo social skills reinforcement through groupbased activities. Adolescents were rewarded with points for displaying positive behaviors to other adolescents during sporting activities (e.g., helping a fellow adolescent, complimenting or praising other adolescents; Sibley et al., 2011). Staff awarded these points to adolescents throughout sessions when they exhibited positive reinforcing behavior towards other adolescents in group discussions and towards parents during combined parent-child sessions. Accumulated points were used towards a weekly prize.

In addition to providing in-vivo social skills reinforcement, recreational activities served as a way to engage adolescents with ADHD, a group that is often difficult to engage in treatment (Liddle, 1995). The recreational activities were utilized (a) as a context in which adolescents could practice problem-solving and social skills, (b) to introduce ideas for group-based activities for adolescents to engage in outside of sessions as many adolescents were inactive at home, and (c) to offer a component that would help adolescents with attentional difficulties "buy into" treatment by allowing them to get outside of the classroom and engage in a less traditional approach to treatment. The latter aligns with the needs of adolescents with ADHD (see Sibley et al., 2011).

Emotion regulation.: BEAM targeted emotion regulation through staff-facilitated group discussions of emotion regulation strategies followed by in-vivo application and practice of emotion regulation strategies during mild stress-induction activities. The purpose of this approach was to help adolescents identify and understand strategies to manage negative emotions, and then to practice using those same strategies in session while experiencing mild negative emotions. Staff and adolescent group members were able to provide feedback on and praise following adolescents' use of emotion regulation strategies. Adolescents completed several emotion regulation exercises, including one where they completed a task while other adolescents attempted to distract or bother them. Adolescents were instructed to build a tower while other adolescents criticized them (under the supervision of staff). This was modeled after the teasing activities utilized with children and puppets in Coping Power (Lochman, Barry, \& Pardini, 2003). Prior to engaging in this task, adolescents learned skills such as diaphragmatic breathing and positive self-talk, to cope with negative emotions.

Emotion regulation was also targeted through a problem-solving framework. Adolescents described problems that they encountered that frustrated them or angered them during the previous week. In line with Dialectical Behavior Therapy (DBT; Linehan, 1993), after acting 
out the scenario, adolescents were asked to identify points where they could have done something differently (Swales, Heard, \& Williams, 2000).

Adolescents also practiced emotion regulation strategies during joint activities with their parents. In one of these activities, adolescents and parents were instructed to build a model out of Lego blocks. However, adolescents were blindfolded and parents were not allowed to touch the blocks. Therefore, parents had to give detailed instructions to their adolescent about what block to pick up and where to place it. This exercise consistently elicited frustration and allowed adolescents to practice emotion regulation strategies together with their parents when frustrated.

Family support.: Adolescents learned problem-solving skills to address and resolve conflicts. Specifically, staff facilitated group discussion on how to identify and describe a problem, list all potential solutions to the problem, and then decide on an amicable resolution that minimized negative consequences and increased positive consequences. This process was adapted from Problem Solving Skills Training (Kazdin, 2010) where youth engage in steps to identify the goal, detail the steps to achieve it, and evaluate the outcome. Following discussion, adolescents practiced this procedure in session using problems elicited from their daily lives. At the end of each session, adolescents joined their parents and practiced these skills together with parents by problem-solving conflict issues present in the parent-child relationship.

Parent component—Given the high heritability of ADHD (Faraone et al., 2005), it was expected that some parents would also exhibit ADHD symptoms. The possibility of parental ADHD symptoms was accommodated by steering away from the traditional didactic model for parent training and instead using a facilitator approach that involved role-playing activities, eliciting participation from parents throughout sessions, and being especially attuned to keeping parents engaged (Chronis, Chacko, Fabiano, Wymbs, \& Pelham, 2004). Consistent with the adolescent component, the parent component of BEAM also consisted of modules targeting reward responsivity, emotion regulation, and family support.

Reward responsivity.: BEAM targeted adolescents' reward responsivity by instructing parents about the links between adolescents' participation in pleasant activities and positive mood. Parents were also instructed in how to use positive reinforcement of their adolescent's engagement in positive activities, with the rationale being that external reinforcers could be used by parents at first to increase the frequency of positive activities and then faded over time as adolescents' anticipatory pleasure increased and the intrinsic enjoyment of positive activities (i.e., consummatory pleasure) reinforced the behavior.

Joint parent-adolescent activities were used to ensure that parents and adolescents had the same understanding as to what activities adolescents found enjoyable and what external reinforcers would be motivating. This was achieved through a modified "Newlywed Game" activity where adolescents secretly wrote down their top activities and top reinforcers and parents tried to match their answers to earn points. Following the game, behavior contracting was utilized to plan adolescent engagement in pleasant activities and establish agreed upon reinforcements for completing planned activities. 
Family support.: BEAM targeted family support by helping parents refine problem-solving skills, improve communication skills, and increase the frequency of positive reinforcement of desirable adolescent behaviors through group discussions and role-plays. For example, parents discussed increasing praise for positive behaviors and increasing frequency of rewards for utilization of emotion-regulation skills to reduce mood problems. They also talked about timing, tone of voice, and active listening to improve communication with their adolescent.

Parents learned problem-solving strategies so as to not engage in arguments with their adolescent and "add fuel to the fire" but rather resolve them systematically in a framework that adolescents also learned in their group sessions (described above in adolescents' "Family Support" section). Parents were given sample scenarios or scenarios offered by parents in group that stemmed from their own personal struggles and together arrived at a potential solution. One area that parents problem-solved was how to successfully balance monitoring their adolescents while still allowing them autonomy. Parents and adolescents came together at the end of session to discuss problems that had arisen during the previous week and, with consultation from clinicians, arrived at solutions for these problems.

Behavior contracting was then used to encourage both adolescents and parents to implement the solutions in the coming week. In combination, these skills were expected to lead to an increase in positive parent-child interactions and thus an increase in family support.

\section{Measures}

Depressive Symptoms-The Children's Depressive Inventory-2 (CDI-2) was used to assess the presence of depressive symptoms in the past 2 weeks. The Children's Depressive Inventory: Parent Version (CDI-P) is a corresponding parent-report version of the CDI-2. On both versions of the CDI-2, raw scores are converted to $T$-scores and $T$-scores above 65 are considered clinically elevated. $T$-scores were used in analyses. In the present sample, coefficient alpha was .82 and .76 for the CDI and CDIP, respectively.

Reward Responsivity-Adolescents' engagement in pleasurable activities was measured using the Tripartite Pleasure Inventory-Hedonic Responsivity subscale (Leventhal, 2012). The TPI describes 12 types of experiences that span interest/pastimes, social interaction, sensory, and goals/mastery (e.g., learning new information or skills, romantic or sexual activities). A mean score is computed resulting in an average ranging from 0 to 4 . In the present sample, coefficient alpha was .86 .

Emotion Regulation-Adolescent self-report of emotion regulation was measured using the Difficulties in Emotion Regulation Scale (DERS; Gratz \& Roemer, 2004). The total score was used in the current study. Previous research in adolescents has indicated the DERS has strong psychometric properties (e.g., Weinberg \& Klonsky, 2009). In the present sample, coefficient alpha was .93. Parent-report of adolescent emotion regulation was measured using the Emotion Regulation Checklist (ERC; Shields \& Cicchetti, 1997), which has been used previously in adolescent samples (e.g., Keiley et al., 2015). As in past research on youth with ADHD and depressive symptoms (Seymour et al., 2012), the lability/negativity factor was used. In the present sample, coefficient alpha was .71. 
Family Support-A disagreement resolution task, adapted from the Family Check-Up (Dishion \& Kavanagh, 2003), was included to measure family support and problem-solving skills. All tasks were video recorded and coded by trained observers. The parent-adolescent dyad recalled an argument or problem that occurred within the last week. The dyad was instructed to spend 10 minutes trying to resolve the argument.

The task was coded using the Interaction Behavior Code (IBC; Prinz \& Kent, 1978) to arrive at the overall problem-solving effectiveness. Raters were first trained on the IBC by having to write the definitions to each behavior code verbatim. Behavior code included a description and examples. After obtaining $100 \%$ on the written portion of training, research assistants coded two training videos where they needed to match all codes against an answer key developed by the principal investigator. Coders met with the principal investigator to review their performance on the first training video before moving on to the second. If necessary, individuals completed the training videos a second time in order to match all codes. At that point, research assistants began coding actual study videos. Though raters were not blind to the purpose of the study, they were blind to the assessment time point. To arrive at problemsolving effectiveness subscale, ratings were completed on a scale of 0 (no), .5 (little), and 1 (yes). The ratings for this variable were separately averaged across raters. The Kappa value for interrater reliability was .78 .

Satisfaction-The Client Satisfaction Questionnaire (CSQ-8; Larsen, Attkisson, Hargreaves, \& Nguyen, 1979) was administered to both parents and adolescents to evaluate participant satisfaction with an intervention and likelihood of recommending it to others.

Adolescents and their parents also completed a semistructured interview at each assessment point following the BEAM treatment program. Participants spoke about their experiences with the preventive intervention including their satisfaction, most helpful modules, BEAM's effectiveness, and suggested modifications. Interviews were transcribed and verified by two research assistants.

Treatment Compliance-Treatment compliance was measured by the rate of attendance at weekly sessions as well as the number of weekly homework assignments completed (i.e., completing daily mood ratings, listing pleasant activities, engaging in pleasant activities, following through with parent-adolescent agreements completed during session).

Treatment Adherence-Parent and child treatment sessions were either audio or video recorded each week. A trained research assistant who was not involved in the provision of treatment evaluated all recordings and completed a check to determine the extent to which staff members adhered to the treatment manual for each session.

\section{Data Analysis}

There was minimal missing data (6.2\%), meaning items that participants failed to answer while completing the assessment battery. Though there was no missing data at pretreatment, there was missing data at all three posttreatment assessment time points. As will be described later, some participants did not complete follow-up assessments. When a participant failed to complete a follow-up assessment, they were excluded from statistical 
analyses at that assessment wave. Little's Missing Completely at Random (MCAR) test was not statistically significant for missing data at posttreatment or either follow-up assessment, consistent with the assumption of data missing completely at random. Missing item-level data were accommodated using multiple imputation (Sinharay, Stern, \& Russell, 2001) averaged across 10 imputation sets. This method has been implemented successfully in small sample research (Graham \& Schafer, 1999).

Paired-samples $T$-tests were conducted on outcome variables (i.e., depressive symptoms, reward responsivity, emotion regulation, and family support) to evaluate whether scores were significantly different at posttreatment and follow-ups as compared to pretreatment. Reliable change indices (RCI; Jacobson \& Truax, 1991) were used to examine individual clinically significant changes in outcome measures (i.e., depressive symptoms, reward responsivity, and emotion regulation). Statistical information necessary for RCI analyses (i.e., test-retest reliability, standard deviations of the normal population) was not available for the family support variable. An RCI greater than or equal to 1.96 indicates reliable change at $p<0.05$.

\section{Results}

\section{Treatment Compliance and Adherence}

All eight families (100\%) attended every BEAM session and completed the posttreatment assessment. Seven (87.5\%) families completed the 6-week follow-up assessment and five (62.5\%) families completed the 12-week follow-up assessment. The mean homework completion percentage across all participants was $58.34 \%$ ( $S D=34.51$, Range $=0-100 \%$ ). Overall adherence for BEAM adolescent sessions and parent sessions was $94 \%$ and $100 \%$, respectively.

\section{Depressive Symptoms and Intervention Targets}

Mean scores on outcome variables at pre, post, and both follow-up assessments are provided in Table 3. Individual scores for outcome variables and results from RCI analyses are presented in Table 1.

Depressive Symptoms-Parent ratings of adolescent depressive symptoms significantly decreased in severity from pre to post $(d=1.46, p=.01)$ and remained significantly lower at the 6-week follow-up as compared to pretreatment $(d=1.02, p=.02)$, but not at the 12-week follow-up. On an individual case level, a majority of parents reported significant decreases in their adolescents' depressive symptoms at posttreatment $(n=5,62.5 \%)$ and the first followup $(n=4,57.1 \%)$. Adolescent self-ratings of depressive symptoms were not significantly lower at the posttreatment, 6-week, 12-week follow-up compared to pretreatment. On an individual case level, a minority of adolescents reported significant changes in depressive symptoms at each of the three posttreatment assessment point $(n=3,37.5 \% ; n=2,28.6 \% ; n$ $=1,20.0 \%$, respectively).

Reward Responsivity-Adolescent self-reported reward responsivity was significantly higher at posttreatment as compared to pretreatment $(d=1.39, p<.001)$. Significant 
increases were not found for reward responsivity at either the 6-weeks or 12-week followups. On an individual case level, a minority of adolescents reported a significant change in reward responsivity from pretreatment to the posttreatment, first follow-up, and second follow-up assessments $(n=3,37.5 \% ; n=2,28.6 \% ; n=2,40.0 \%$, respectively).

Emotion Regulation-Parent ratings of adolescent emotion dysregulation levels significantly decreased from pre to post $(d=0.86, p=.03)$ and remained significantly lower at 6-week follow-up as compared to pretreatment $(d=0.89, p=.004)$, but not at the 12-week follow-up. On an individual case level, a majority of parents reported a significant decrease compared to pretreatment in emotion dysregulation at posttreatment $(n=6,62.5 \%)$ and both follow-ups $(n=5,85.7 \% ; n=3,60.0 \%)$. Adolescent self-ratings of emotion regulation levels did not significantly differ at posttreatment or at the 6-week and 12-week follow-up assessments compared to pretreatment. On an individual case level, a minority of adolescents reported a significant increase in emotion regulation at posttreatment $(n=1$, $12.5 \%)$ and the second follow-up $(n=1,20.0 \%)$, and a majority reported a significant increase at the first follow-up compared to pretreatment $(n=4,57.1 \%)$.

Family Support-Parent-adolescent dyads' problem-solving effectiveness did not significantly increase at posttreatment, 6-week, or the 12-week follow-up assessments.

\section{Intervention Satisfaction}

Adolescents and parents reported high satisfaction with BEAM; CSQ scores for adolescents and parents were $26.13(S D=5.22)$ and $26.63(S D=5.31)$, respectively. In semistructured interviews, parents and adolescents generally expressed a positive appraisal of the BEAM session schedule. Parents reported that having BEAM sessions on Saturday morning was "the only way I can fit it." A majority of parents in attendance were working mothers and fathers, so having sessions after parents and adolescents arrived home from work and school would have presented logistical barriers to session attendance.

In addition to holding sessions on Saturday mornings, parents expressed enthusiasm about the brief, four-session format. Parents and adolescents expressed that having only four sessions kept the material "structured" and "tight" and required less commitment than traditional treatment programs.

The group format of BEAM was well-received by all eight families. Parents found that the group format created a sense of community much like a support group. Discussing issues with their adolescent's mood and receiving feedback from other parents was experienced as validating, and parents reported feeling at ease discussing family issues in the group setting. For example, one mother stated that the group format helped her realize "We're not alone, our kids have different types of issues, but we're all kind of in the same boat, trying to figure them out. I get a lot out of that personally.... Whenever I'm in a room with people that understand me, it just reenergizes me." Adolescents also viewed the group format favorably, as evidenced by statements such as being in a group setting made it "easier to make friends" and "helped ... know how the teens felt like ... how they were doing at home and school." The active dialogue between parents and the facilitator, rather than a lecture format, also kept parents engaged. One mother stated, "We didn't have any opportunity to get bored." 
The BEAM material was helpful and presented clearly. Parents responded positively to the material covered in BEAM. One mother stated that the program “... was amazing because I can ... deal with her condition a little more or easier ... [I learned] strategies to help her through her issues and I think it was good, it helped me and helped her." Another mother "liked that we list different ideas and tasks that we can do with our kids because sometimes you just get in a rut and you just go to that one, that one task and that one routine and that's it." Parents also found the workbooks user friendly and liked how session content in the parent group overlapped with the content in the adolescent group: "She's getting the same instructions that I'm getting so when I say to her that we've got to do this, it's easier to get going because we're both playing by the same rules. So I think the materials were spot on." Adolescents overwhelmingly commented positively on the content of BEAM. They reported liking learning the skills through "real life problems ... or situations" and through sports activities where "there was a lot of communication and everybody would help each other." Furthermore, adolescents reported the material was presented clearly and explained well.

In regard to specific skills, the downward and upward spiral discussion was cited as the most widely used skill by parents. For example, one mother indicated "I'm able to discern faster where the point of no return is." Another mother illustrated the usefulness of the spirals further: "What I never had learned was to stop. If I see her going into a really dark place.... You guys taught me there is a moment that you have to just stop and walk away because if not you force them into a darker place or a darker kind of mindset. And now that's really deliberate. I either stop or I find a way to just shift focus really fast." Pleasant activity scheduling was cited as the most widely used skill by adolescents. For example, one adolescent said, "I did that all the time, literally every time I got upset, I tried to do that." Adolescents reported engaging in a variety of pleasant activities, including drawing, playing sports, reading a book, building Legos, playing with pets, listening to music, and cooking.

A reduction in mood problems was the most commonly cited improvement by parents. More specifically, parents reported that the adolescents were "less stressed," "not as irritable" and "less moody." After BEAM, one parent expressed a desire for her son to taper and discontinue mood medication because she believed BEAM led to stable improvements in his mood. Adolescents also reported fewer problems regarding their mood during interviews. For example, when asked about their mood and stress levels, adolescent responses included, "I'm happier," "a lot less depression," "I haven't been really getting angry," "I don't get mad as much," "I haven't had that much stress like I used to," "and "I'm a lot less stressed." Improvements in communication and the parent-child relationship were reported by both parents and adolescents. Following BEAM, parents reported that their adolescents were "more open to feedback," there was more open communication in the household, and there was better communication regarding each other's feelings and fewer arguments. One mother stated that granting more autonomy helped improve her relationship with her son: "Letting him earn more time on his own going out, whatever, and I think allowing him to do that made me feel more comfortable and made him ... feel like he is growing up and more confident and I think that was good ... [he loves] to do things on his own and every time he does it, I feel more relaxed." 
Adolescents also reported improvements in their relationships with their parents. For example: "We're talking more now. She doesn't get mad that often," and "she's more likely to listen to my side of the story."

\section{Suggestions for Improving Intervention}

In semistructured interviews, parents and adolescents made several constructive suggestions that will inform revisions to BEAM. The first suggestion voiced was regarding homework. Parents requested an increased number of structured homework assignments. This is somewhat surprising given that majority of adolescent participants completed two thirds or less of assigned homework. This rate of homework completion is not unique to BEAM (e.g., Gaynor, Lawrence, \& Nelson-Gray, 2006; Sibley et al., 2013), but increasing homework completion represents a priority for future work. Adolescents may have partially completed homework assignments (e.g., pleasant activities) but may not have been tracking their mood in their mood journal. An Internet or cell phone application-based mood tracker may make completing mood journals easier. Internet-based applications are gaining traction in mental health (Luxton, June, \& Kinn, 2011) and represent one strategy to make BEAM homework assignments more palatable to adolescents.

A second suggestion was building in more opportunities for interaction and networking with other parents and adolescents following BEAM. For example, one parent expressed wanting a system by which parents could exchange names and phone numbers so that their adolescents could continue spending time together after the program. One mother said in regard to the adolescents in BEAM: "They build a relationship with the other kids and then it's like they are yanked away from each other. And I think these kids already have difficulties bonding with other peers and when they actually make a bond to have it yanked away like that it's a little traumatic." Adolescents also reported wanting more time to socialize and talk with the other adolescents. For example, one adolescent suggested having a lunch time with the adolescents to increase time available to socialize and talk more amongst each other.

Parents suggested that after BEAM, review sheets should be provided. Though an adolescent and a parent workbook were provided to each family that they could keep following BEAM, one mother suggested sending out "bullet points of everything you've done, like all the strategies." Perhaps alongside workbooks, a treatment summary could be provided.

\section{Illustrative Clinical Vignettes}

Two cases (Family 1 and Family 8) are described below to illustrate BEAM on individual case levels.

\section{Family 1}

The first parent-adolescent dyad consisted of a 13-year-old Hispanic female in $8^{\text {th }}$ grade and her mother. The mother indicated that her daughter's "massive mood swings" prompted their participation in BEAM. Prior to treatment, the mother reported clinically elevated levels of adolescent depressive symptoms $(T=71)$ and the daughter reported average levels of depressive symptoms $(T=55)$. The mother and adolescent attended all four sessions of 
BEAM and the adolescent completed $100 \%$ of the weekly homework assignments. Following BEAM, the family completed the posttreatment and both follow-up assessments. Though the mother reported having difficulties with convincing the adolescent to attend sessions, she reported that her daughter's resistance was reduced when she explained how BEAM was just as much for her (the mother) as it was for the daughter.

Though the adolescent was relatively quiet during group discussions, her contributions were on-task and constructive. During review of weekly mood journals, the adolescent reported using activities like drawing and skateboarding to elevate her mood. During a frustration task in Session 3, the adolescent reported using positive self-talk (i.e., "I can do this") to regulate her emotions. The adolescent enjoyed recreational activities and assumed a leadership role during those activities by passing the ball to other players (even those who may not have possessed the same technique as her) and appropriately explaining rules to others. The adolescent frequently earned points toward weekly prizes by exhibiting this prosocial behavior. Staff played the sports alongside adolescents so that they could be consistently praising and awarding points (positively reinforcing) for these behaviors with the goal of increasing social skills.

The mother was actively engaged in sessions. In particular, the mother spoke to the group about difficulty letting her daughter be more independent. For example, she shared that her daughter frequently asked to walk to a fast food restaurant close to her school when classes let out. The mother refused to let her go to the restaurant because she believed the daughter would lose track of time and be late getting back to the school's after-care program. (She noted her daughter was late for school multiple times each week.) Another mother suggested that she allow her adolescent to earn the privilege of going to the restaurant by arriving on time for school a certain number of days during the week. Other parents similarly encouraged her to allow her daughter to earn her independence by proving that she can be on time. Following conversations with other parents and the facilitator about appropriate monitoring while fostering her daughter's independence as well as using rewards for positive behavior, her mother agreed to allow her daughter to go to the restaurant after school if she got to school on time.

The mother found the communication skills (Lego) activity during Session 2 to be beneficial. For her, it was eye opening when she realized she was not listening to instructions and therefore gave incorrect instructions to her daughter. She did not realize the model had to not only be built according to the shape on the card but also color coded, as well. She stated, "I heard something, but it wasn't what I was supposed to be doing so we had to knock it down and start all over again. So I thought that was really good to help us understand how to listen better."

The parent and the adolescent expressed high satisfaction with the program as evidenced by the mother stating, "You guys did a great job and I'm happy with overall everything that I've gotten out of [your program]." The adolescent noted that the program helped improve her relationship with her mother as she was not getting as angry with her and they were able to resolve their problems in a healthier manner. 


\section{Family 8}

The second parent-adolescent dyad consisted of a 13-year-old, Hispanic male in $7^{\text {th }}$ grade and his mother. During the phone screen, both mother and adolescent reported depressive symptoms in the clinical range (adolescent $T=67$, parent $T=69$ ). The mother indicated that her son's behavior was often destructive, and that he had been psychiatrically hospitalized for emotional outbursts and aggressive behaviors twice. She hoped that BEAM would help them learn how to prevent these future emotional outbreaks and hospitalizations. The parent and adolescent dyad attended all sessions of BEAM. The adolescent completed two thirds of homework assigned. They completed the posttreatment and both follow-up assessments.

Throughout BEAM sessions, the adolescent was excessively talkative and often hyperactive (e.g., inter-rupting others and blurting out answers, fidgeting in his seat and playing with his belongings during classroom-based activities). Counselors ignored his mildly disruptive behavior and praised him for being on task and sitting appropriately. During one session, the adolescent became argumentative with staff after they did not allow him to leave session to get a coffee. Counselors did not give in to his demands and he did not earn tickets during the activities that he was disrupting. He refused to participate in the recreational activity so the adolescent sat on the sidelines and staff monitored him while ensuring they were not giving him attention for his misbehavior.

The active, hands-on approach to sessions was a good match for his hyperactive behavior. Having a recreation time where the adolescent could be active and play sports with his peers "made everything not dull," helped break up the session, and held his attention more. The adolescent also reported not engaging in recreational activities at home so this component of BEAM introduced ideas for pleasant activity scheduling. The adolescent seemed to enjoy the recreational activities given that he suggested they be longer during his posttreatment interview. When generating ideas for pleasant activities, he indicated that viable social options would include spending time with his siblings playing Legos or playing with the cat. Practicing guitar and piano emerged as productive activities. The adolescent expressed interest in using video games as a pleasant activity. The pros (it was an enjoyable activity for him) and cons (it was neither social nor productive) were discussed and ultimately the adolescent removed this activity from his list.

The adolescent also proved to be an asset to the group during problem-solving discussions. He provided a definition for problem solving (i.e., "finding an obstacle and removing it"), encouraged his peers to calmly define the problem and to see the other person's side of the argument, and listed numerous solutions to a hypothetical problem proposed. He also helped eliminate solutions that were "possible but not plausible."

The adolescent enacted problem solving as a skill outside of sessions. At the follow-up assessment, his mother reported, "It's mostly the negotiating we do a lot of ... he actually said, 'Let's set up a contract, let's figure it out, let's negotiate' ... I think that what he's doing is that he's setting himself some goals that can't set on his own. It's like, 'I can't set them up on my own, you help me set them up.' And I think that's a valid thing."

Cogn Behav Pract. Author manuscript; available in PMC 2019 November 29. 
The mother and adolescent reported finding the program helpful. In the first follow-up assessment she explained, "He hasn't gotten angry. Or he hasn't gotten depressed." She also reported being more attuned to early signs of her son shifting into a negative mood and intervening to distract him: "The other thing that I try to be is very aware [when he's going into a downward spiral] ... When he's starting to go like that I drive him to the gym with me. So he'll go on the treadmills." When asked generally about her experience in BEAM, she stated, "It's easy to learn how to do the right thing and then forget how to do the right thing ... I mean you go back to being on task so that the bad times become more sporadic and the good times become more frequent. So that's the one thing I liked. It's hard to be a parent to a kid that's explosive ... You just have to stop the triggers. ... We're doing better."

\section{Discussion}

Findings from this open trial of the BEAM depression preventive intervention for adolescents with ADHD provide strong preliminary support for the feasibility of BEAM, as $100 \%$ of families attended the four intervention sessions, and it was delivered with high adherence. The perfect attendance in this open trial of BEAM compares favorably to dropout rates in parent training programs for externalizing disorders (ranging from $28 \%$ to $50 \%$; Reyno \& McGrath, 2006) and parent-adolescent treatment groups for depression (e.g., 10\% dropout in the Coping with Depression program; Lewinsohn, Clarke, Hops, \& Andrews, 1990). Findings also provide support for patient satisfaction, as parents and adolescents expressed high satisfaction with BEAM. In terms of primary outcome and intervention targets, preliminary support was found for targeted changes in depressive symptoms and emotion regulation at posttreatment and the 6-week follow-up via parent ratings and reward responsivity at posttreatment via adolescent ratings.

Although results overall were supportive of BEAM, there were often discrepancies between parent and adolescent ratings on outcome variables. For example, a majority of parents rated clinically significant decreases in their adolescents' depressive symptoms at posttreatment and the first follow-up compared to pretreatment, whereas a majority of adolescents rated no significant changes in depressive symptoms at any assessment point. The discrepancy in parent and adolescent ratings of depressive symptom severity is not uncommon (e.g., Achenbach et al., 2003; de Los Reyes \& Kazdin, 2005) and may be due in part to the tendency of adolescents with ADHD to underestimate the severity of their own symptoms and impairment (Sibley et al., 2010). The present findings are consistent with this possibility, in that adolescent-rated depressive symptoms were considerably lower than parent-rated depressive symptoms at the pretreatment intake assessment. This suggests there may have been a floor effect on adolescent self-ratings which limited the ability to detect intervention effects. In regard to depressive symptoms, it is unclear whether parents or adolescents are more accurate informants in general (see Klein, Dougherty, \& Olino, 2005), although the tendency of adolescents with ADHD to underreport symptoms and impairment might indicate parent ratings are preferable in this population. Alternatively, the lack of adolescent-rated change in depressive symptoms and other outcome variables might also have resulted from an insufficient dose of treatment within the 4 BEAM sessions or the short follow-up period. Previous depression prevention research has indicated changes in 
outcomes may be seen after several months (Horowitz \& Garber, 2006). Future trials of the BEAM program should monitor outcomes beyond 3 months posttreatment.

As a group treatment, parents and adolescents interacted with one another throughout the course of BEAM. This format was frequently and favorably mentioned in the qualitative interviews. The prosocial aspect of BEAM may have contributed to changes in outcome variables, meaning that positive social interactions with peers during treatment may have contributed to positive outcomes seen at posttreatment. Further, the cessation of such positive social interactions after treatment may have contributed to the absence of additional positive outcomes seen at follow-up. However, we did not directly measure peer social support and positive social interactions in the current study.

We selected participants for this study primarily on the basis of subthreshold depressive symptoms. This is consistent with selection strategies commonly used in depressive prevention approaches and is supported by extensive empirical evidence on cut scores for depressive symptoms in adolescents. An interesting topic for future research would be to examine the potential for participant screening and selection based on mediator variables rather than depressive symptoms (i.e., selection based on intervention targets rather than the intervention outcome).

Though parents and adolescents reported positive effects and satisfaction with BEAM, they also offered several suggestions for improving the delivery of BEAM. These included increasing the number of structured homework assignments to practice skills, using Internet or cell-phone based technology to complete homework and track progress, increasing opportunities for parents and adolescents to network outside of session, and providing parents and teens with BEAM program review sheets.

The present study's findings should be interpreted in light of its strengths and limitations. Strengths include the use of multiple informants, collection of data through the use of psychosocial rating scales and behavior observations, and the use of semistructured interviews to determine diagnostic histories, and a sample with high ethnic minority representation. The BEAM program was also designed to be implemented easily by mental health professionals with varying degrees of expertise in various community settings, such as schools. Limitations include the small sample and low statistical power to detect effects, as well as the absence of a control group in this pilot open trial. It is unknown the extent to which improvements from pretreatment to posttreatment and follow-ups can be attributed to BEAM or to other factors such as regression to the mean, maturation, testing effects, and so on. The changes seen following the BEAM program could have been a booster or augmentation of the previous behavioral treatment families received. For example, the review of behavioral principles for ADHD in the parent group and the classroom-like setting may have reinstated previously learned strategies for treating ADHD. Further, it is possible that parent-rated changes in adolescent outcomes may have also been influenced by positive effects of BEAM on parents' own mental health; this possibility may also account for discrepancies in parent and adolescent ratings. Future work should examine the influence of BEAM on parents' levels of depressive symptoms. 
One of the requirements for participation in BEAM was parents had to have previously completed behavioral treatment for ADHD to ensure parents would have a basic understanding of behavioral treatment principles. Therefore, the severity of ADHD symptoms in our sample may have been less than that of untreated adolescents with ADHD. This may limit the generalizability of findings based on ADHD symptom severity and presence or intensity of past behavioral treatments. The current study did not measure ADHD symptoms or global functioning at pre- and posttreatment. Future work should examine how baseline ADHD symptoms and intensity of treatment may affect changes in depressive symptoms and mediator variables following BEAM. In a similar vein, it is worth exploring the contributions that early intervention and/or continued treatment for ADHD would have on reducing the risk of depression. Specifically, targeting reductions in functional impairment directly related to ADHD may represent another promising means to prevent subsequent depression.

Though parents and adolescents were encouraged and did provide constructive criticisms of BEAM during their qualitative interviews, the fact that one of the treatment providers conducted the interviews may have resulted in families being less comfortable or willing to provide criticisms. In addition to a control group, it will be important for future studies to use independent evaluators in the assessment of quantitative and qualitative outcomes.

In summary, this report has provided an overview of the development and implementation of the BEAM depression prevention program for adolescents with ADHD. Families were highly satisfied with BEAM and staff reported the program was easy to implement. Findings were promising with respect to reducing depressive symptoms, at least according to parent ratings, and suggest the BEAM program moved the needle on intervention targets (i.e., adolescent-reported reward responsivity, parent-reported emotion regulation). Findings need to be interpreted in light of discrepancies between parent and adolescent ratings on outcome measures, with more promising findings coming from parent ratings than adolescent selfratings. Overall, these findings support a move to the next stage of intervention development, a randomized control trial of the BEAM program.

\section{References}

Achenbach TM, Dumenci L, \& Rescorla LA (2003). DSM-oriented and empirically based approaches to constructing scales from the same item pools. Journal of Clinical Child and Adolescent Psychology, 32(3), 328-340. 10.1207/S15374424JCCP3203_02 [PubMed: 12881022]

Anastopoulos AD, Smith TF, Garrett ME, Morrissey-Kane E, Schatz NK, Sommer JL, ... AshleyKoch A (2011). Self-regulation of emotion, functional impairment, and comorbidity among children with AD/HD. Journal of Attention Disorders, 15(7), 583-592. 10.1177/1087054710370567 [PubMed: 20686097]

Angold A, Costello EJ, \& Erkanli A (1999). Comorbidity. Journal of Child Psychology and Psychiatry, 40(1), 57-87. [PubMed: 10102726]

Biederman J, Ball SW, Monuteaux MC, Mick E, Spencer TJ, McCreary M, ... Faraone SV (2008). New insights into the comorbidity between ADHD and major depression in adolescent and young adult females. Journal of the American Academy of Child and Adolescent Psychiatry, 47(4), 426434. 10.1097/CHI.0b013e31816429d3 [PubMed: 18388760]

Biederman J, Newcorn J, \& Sprich S (1991). Comorbidity of attention deficit hyperactivity disorder with conduct, depressive, anxiety, and other disorders. American Journal of Psychiatry, 148(5), 564577. [PubMed: 2018156]

Cogn Behav Pract. Author manuscript; available in PMC 2019 November 29. 
Bogdan R, \& Pizzagalli DA (2009). The heritability of hedonic capacity and perceived stress: A twin study evaluation of candidate depressive phenotypes. Psychological Medicine, 39(2), 211-218. 10.1017/S0033291708003619 [PubMed: 18507876]

Chronis AM, Chacko A, Fabiano GA, Wymbs BT, \& Pelham WE (2004). Enhancements to the behavioral parent training paradigm for families of children with ADHD: Review and future directions. Clinical Child and Family Psychology Review, 7(1), 1-27. [PubMed: 15119686]

Claude D, \& Firestone P (1995). The development of ADHD boys: A 12-year follow-up. Canadian Journal of Behavioural Science, 27(2), 226-249.

Cunningham CE, \& Barkley RA (1979). The interactions of normal and hyperactive children with their mothers in free play and structured tasks. Child Development, 50(1), 217-224. [PubMed: 446207]

de Los Reyes A, \& Kazdin AE (2005). Informant discrepancies in the assessment of childhood psychopathology: A critical review, theoretical framework, and recommendations for further study. Psychological Bulletin, 131(4), 483-509. [PubMed: 16060799]

Dimidjian S Barrera M Jr, Martell C, Munoz RF \& Lewinsohn PM (2011). The origins and current status of behavioral activation treatments for depression. Annual Review of Clinical Psychology, 7,1-38. 10.1146/annurev-clinpsy-032210-104535

Dishion TJ, \& Kavanagh K (2003). Intervening in adolescent problem behavior: A family-centered approach. New York: Guilford Press.

Durbin C, \& Shafir D (2008). Emotion regulation and risk for depression In Abela JRZ \& Hankin BL (Eds.), Handbook of depression in children and adolescents (pp. 149-176). New York: Guilford.

Durston S (2003). A review of the biological bases of ADHD: What have we learned from imaging studies? Mental Retardation and Developmental Disabilities Research Reviews, 9(3), 184-195. 10.1002/mrdd.10079 [PubMed: 12953298]

Faraone SV, Perlis RH, Doyle AE, Smoller JW, Goralnick JJ, Holmgren MA, \& Sklar P (2005). Molecular genetics of attention-deficit/hyperactivity disorder. Biological Psychiatry, 57(11), 13131323. doi: S0006-3223(04)01226-0. [PubMed: 15950004]

Feng X, Keenan K, Hipwell AE, Henneberger AK, Rischall MS, Butch J, .. Babinski DE (2009). Longitudinal associations between emotion regulation and depression in preadolescent girls: Moderation by the caregiving environment. Developmental Psychology, 45(3), 798-808. 10.1037/ a0014617 [PubMed: 19413432]

Gaynor ST, Lawrence PS, \& Nelson-Gray RO (2006). Measuing homework compliance in cognitivebehavioral therapy for adolescent depression. Behavior Modification, 30(5), 647-672. [PubMed: 16894234]

Graham JW, \& Schafer JL (1999). On the performance of multiple imputation for multivariate data with small sample size In Hoyle RH (Ed.), Statistical strategies for small sample sizes (1-29). Thousand Oaks, CA: Sage Publications.

Gratz K, \& Roemer L (2004). Multidimensional assessment of emotion regulation and dysregulation: Development, factor structure, and intial validation of the difficulties in emotion regulation scale. Journal of Psychopathology and Behavioral Assessment, 26(1), 41-54.

Hopko DR, Lejuez CW, Ruggiero KJ, \& Eifert GH (2003). Contemporary behavioral activation treatments for depression: Procedures, principles, and progress. Clinical Psychology Review, 23(5), 699-717. [PubMed: 12971906]

Horowitz JL, \& Garber J (2006). The prevention of depressive symptoms in children and adolescents: A meta-analytic review. Journal of Consulting and Clinical Psychology, 74(3), 401-415. [PubMed: 16822098]

Humphreys KL, Katz SJ, Lee SS, Hammen C, Brennan PA, \& Najman JM (2013). The association of ADHD and depression: Mediation by peer problems and parent-child difficulties in two complementary samples. Journal of Abnormal Psychology, 122(3), 854-867. 10.1037/a0033895 [PubMed: 24016021]

Jacobson NS, \& Truax P (1991). Clinical significance: a statistical approach to defining meaningful change in psychotherapy research. Journal of Consulting and Clinical Psychology, 59(1), 12-19. [PubMed: 2002127] 
Jensen JB, Burke N, \& Garfinkel BD (1988). Depression and symptoms of attention deficit disorder with hyperactivity. Journal of the American Academy of Child and Adolescent Psychiatry, 27(6), 742-747. doi: S0890-8567(09)65856-1. [PubMed: 3198561]

Johnston C (1996). Parent characteristics and parent-child interactions in families of nonproblem children and ADHD children with higher and lower levels of oppositional-defiant behavior. Journal of Abnormal Child Psychology, 24(1), 85-104. [PubMed: 8833030]

Kato T (2007). Molecular genetics of bipolar disorder and depression. Psychiatry and Clinical Neurosciences, 61(1), 3-19. 10.1111/j.1440-1819.2007.01604.x [PubMed: 17239033]

Kazdin AE (2010). Problem-solving skills training and parent management training for opposition defiant disorder and conduct disorder In Weisz JR \& Kazdin AE (Eds.), Evidence based pychotherapies for children and adolescents (2nd ed., pp. 211-226). New York: Guilford Press.

Keiley MK, Zaremba-Morgan A, Datubo-Brown C, Pyle R, \& Cox M (2015). Multiple-family group intervention for incarcerated male adolesents who sexually offend and their families: Change in maladaptive emotion regulation predicts adaptive change in adolescent behaviors. Journal of Marital and Family Therapy, 41(3), 324-339. [PubMed: 24809985]

Klein DN, Dougherty LR, \& Olino TM (2005). Toward guidelines for evidence-based assessment of depression in children and adolescents. Journal of Clinical Child and Adolescent Psychology, 34(3), 412-432. 10.1207/s15374424jccp3403_3 [PubMed: 16026212]

Kovacs M (2011). Children's Depression Inventory 2 (CDI 2) (2nd ed.). North Tonawanda, NY: MultiHealth Systems Inc.

Kuyken W, Watkins E, Holden E, \& Cook W (2006). Rumination in adolescents at risk for depression. Journal of Affective Disorders, 96(1-2), 39-47. [PubMed: 16837053]

Larsen DL, Attkisson CC, Hargreaves WA, \& Nguyen TD (1979). Assessment of client/patient satisfaction: Development of a general scale. Evaluation and Program Planning, 2(3), 197-207. [PubMed: 10245370]

Leventhal AM (2012). Relations between anhedonia and physical activity. American Journal of Health Behavior, 36(6), 860-872. [PubMed: 23026043]

Lewinsohn PM (1974). A behavioral approach to depression In Friedman RJ \& Katz MM (Eds.), The psychology of depression: Contemporary theory and research (pp. 157-187). New York: Wiley.

Lewinsohn PM, Clarke GN, Hops H, \& Andrews J (1990). Cognitive-behavioral treatment for depressed adolescents. Behavior Therapy, 21(4), 385-401.

Liddle HA (1995). Conceptual and clinical dimensions of a multidimensional, multisystems engagement strategy in family-based treatment. Psychotherapy, 32(1), 39-58.

Linehan MM (1993). Skills training manual for treating borderlie personality disorder. New York: Guiford Press.

Lochman JE, Barry TD, \& Pardini DA (2003). Anger control training for aggressive youth In Kazdin AE \& Weisz JR (Eds.), Evidence-based psychotherapies for children and adolescents. New York: Guilford Press.

Luxton DD, June JD, \& Kinn JT (2011). Technology-based suicide prevention: Current applications and future directions. Telemedicine Journal and E-Health, 17(1), 50-54. [PubMed: 21214382]

Mannuzza S, Klein RG, Bessler A, Malloy P, \& LaPadula M (1998). Adult psychiatric status of hyperactive boys grown up. American Journal of Psychiatry, 155(4), 493-498. [PubMed: 9545994]

Mannuzza S, Klein RG, Bonagura N, Malloy P, Giampino TL, \& Addalli KA (1991). Hyperactive boys almost grown up: V. Replication of Psychiatric Status. Archives of General Psychiatry, 48(1), 77-83. [PubMed: 1984764]

Mazzuchelli T, Kane R, \& Rees C (2009). Behavioral activation treatments for depression in adults: A meta-analysis and review. Clinical Psychology: Science and Practice, 16(4), 383-411.

McCauley E, Schloredt K, Gudmundsen G, Martell C, \& Dimidjian S (2011). Expanding behavioral activation to depressed adolescents: Lessons learned in treatment development. Cognitive and Behavioral Practice, 18, 371-383.

Meinzer MC, Hill RM, Pettit JW, \& Nichols-Lopez KA (2014). Parental support partially accounts for the covariation between ADHD and depressive symptoms in college students. Journal of Psychopathology and Behavioral Assessment, 37, 247-255. 
Meinzer MC, Lewinsohn PM, Pettit JW, Seeley JR, Gau JM, Chronis-Tuscano A, \& Waxmonsky JG (2013). Attention-deficit/hyperactivity disorder in adolescence predicts onset of major depressive disorder through early adulthood. Depression and Anxiety, 30(6), 546-553. 10.1002/da.22082 [PubMed: 23424020]

Meinzer MC, Pettit JW, Leventhal AM, \& Hill RM (2012). Explaining the covariance between attention-deficit hyperactivity disorder symptoms and depressive symptoms: the role of hedonic responsivity. Journal of Clinical Psychology, 68(10), 1111-1121. [PubMed: 22777931]

Meinzer MC, Pettit JW, \& Viswesvaran C (2014). The co-occurrence of attention-deficit/hyperactivity disorder and unipolar depression in children and adolescents: A meta-analytic review. Clinical Psychology Review, 34(8), 569-607.

Melnick SM, \& Hinshaw SP (2000). Emotion regulation and parenting in AD/HD and comparison boys: Linkages with social behaviors and peer preference. Journal of Abnormal Child Psychology, 28(1), 73-86. [PubMed: 10772351]

Molina BSG, Hinshaw S, Swanson J, Arnold L, Vitiello B, Jensen P, ... MTA Cooperative Group. (2009). The MTA at 8 years: Prospective follow-up of children treated for combined-type ADHD in a multisite study. Journal of the American Academy of Child and Adolescent Psychiatry, 48(5), 484-500. [PubMed: 19318991]

Norvilitis JM, Casey RJ, Brooklier KM, \& Bonello PJ (2000). Emotion appraisal in children with attention deficit/hyperactivity disorder and their parents. Journal of Attention Disorders, 4(1), 1526. $10.1177 / 108705470000400102$

Prinz RJ, \& Kent RN (1978). Recording parent-adolescent interactions without use of frequency or interval-by-interval coding. Behavior Therapy, 9(4), 602-604.

Reyno SM, \& McGrath PJ (2006). Predictors of parent training efficacy for child externalizing behavior problems: A meta-analytic review. Journal of Child Psychology and Psychiatry, 47(1), 99-111. 10.1111/j.1469-7610.2005.01544.x [PubMed: 16405646]

Ritschel LA, Ramirez CL, Jones M, \& Craighead WE (2011). Behavioral activation for depressed teens: A pilot study. Cognitive and Behavioral Practice, 18, 281-299.

Scheres A, Milham MP, Knutson B, \& Castellanos FX (2007). Ventral striatal hyporesponsiveness during reward anticipation in attention-deficit/hyperactivity disorder. Biological Psychiatry, 61 (5), 720-724 (doi: S0006-3223(06)00588-9). [PubMed: 16950228]

Seymour KE, Chronis-Tuscano A, Halldorsdottir T, Stupica B, Owens K, \& Sacks T (2012). Emotion regulation mediates the relationship between ADHD and depressive symptoms in youth. Journal of Abnormal Child Psychology, 40(4), 595-606. [PubMed: 22113705]

Seymour KE, Chronis-Tuscano A, Iwamoto DK, Kurdziel G, \& Macpherson L (2014). Emotion regulation mediates the association between ADHD and depressive symptoms in a community sample of youth. Journal of Abnormal Child Psychology, 42(4), 611-621. [PubMed: 24221724]

Shields A, \& Cicchetti D (1997). Emotion regulation among school-age children: the development and validation of a new criterion Q-sort scale. Developmental Psychology, 33(6), 906-916. [PubMed: 9383613]

Sibley MH, Pelham WE, Derefinko KJ, Kuriyan AB, Sanchez F, \& Graziano PA (2013). A pilot trial of Supporting teens' academic need's daily (STAND): A parent-adolescent collaborative intervention for ADHD. Journal of Psychopathology and Behavioral Assessment, 35, 436-449.

Sibley MH, Pelham WE, Evans SW, Gnagy EM, Ross JM, \& Griener AR (2011). An evaluation of a summer treatment program for adolescents with ADHD. Cognitive and Behavioral Practice, 18, 530-544.

Sibley MH, Pelham WE, Molina BS, Waschbusch DA, Gnagy EM, Babinski DE, \& Biswas A (2010). Inconsistent self-report of delinquency by adolescents and young adults with ADHD. Journal of Abnormal Child Psychology, 38(5), 645-656. [PubMed: 20309624]

Sinharay S, Stern HS, \& Russell D (2001). The use of multiple imputation for the analysis of missing data. Psychological Methods, 6(4), 317-329. 10.1037//1082-989X.6.4.317 [PubMed: 11778675]

Smith BH, Barkley RA, \& Shapiro CJ (2006). Attention-deficit/hyperactivity disorder In Mash EJ \& Barkley RA (Eds.), Treatment of childhood disorders (3rd ed., pp. 65-136). New York: Guilford Press. 
Stice E, Shaw H, Bohon C, Marti CN, \& Rohde P (2009). A meta-analytic review of depression prevention programs for children and adolescents: Factors that predict magnitude of intervention effects. Journal of Consulting and Clinical Psychology, 77(3), 486-503. [PubMed: 19485590]

Swales M, Heard HL, \& Williams JMG (2000). Linehan's Dialectical Behaviour Therapy (DBT) for borderline personality disorder: Overview and adaption. Journal of Mental Health, 9(1), 7-23.

Walcott CM, \& Landau S (2004). The relation between disinhibition and emotion regulation in boys with attention-deficit/hyperactivity disorder. Journal of Clinical Child and Adolescent Psychology, 33(4), 772-782. [PubMed: 15498744]

Weinberg A, \& Klonsky E (2009). Measurement of emotion dysregulationin adolescents. Psychological Assessment, 21(4), 616-621. [PubMed: 19947794]

Wells KC, Chi TC, Hinshaw SP, Epstein JN, Pfiffner L, Nebel-Schwalm M, ... Wigal T (2006). Treatment-related changes in objectively measured parenting behaviors in the multimodal treatment study of children with attention-deficit/hyperactivity disorder. Journal of Consulting and Clinical Psychology, 74(4), 649-657. [PubMed: 16881772]

Wolraich ML, Wibbelsman CJ, Brown TE, Evans SW, Gotlieb EM, Knight JR, ... Wilens T (2005). Attention-deficit/hyperactivity disorder among adolescents: a review of the disorder among adolescents: A review of the diagnosis, treatment, and clinical implications. Pediatrics, 115(6), 1734-1746. [PubMed: 15930238]

Wood AC, \& Neale MC (2010). Twin studies and their implications for molecular genetic studies: Endophenotypes integrate quantitative and molecular genetics in ADHD research. Journal of the American Academy of Child and Adolescent Psychiatry, 49(9), 874-883. 10.1016/j.jaac. 2010.06.006 [PubMed: 20732624]

Zeiss AM, Lewinsohn PM, \& Munoz RF (1979). Nonspecific improvement effects in depression using interpersonal skills training, pleasant activity schedules, or cognitive training. Journal of Consulting and Clinical Psychology, 47(3), 427-439. [PubMed: 528711] 


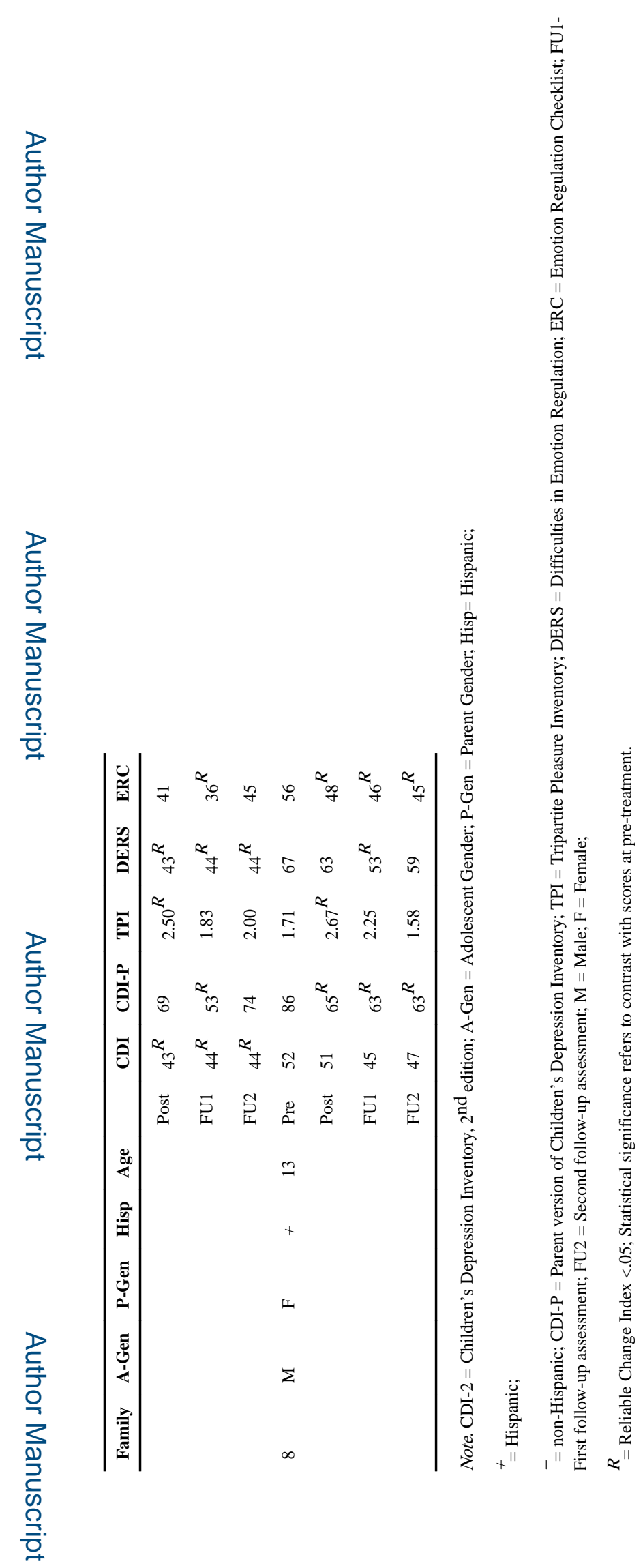

Cogn Behav Pract. Author manuscript; available in PMC 2019 November 29. 


\section{를}
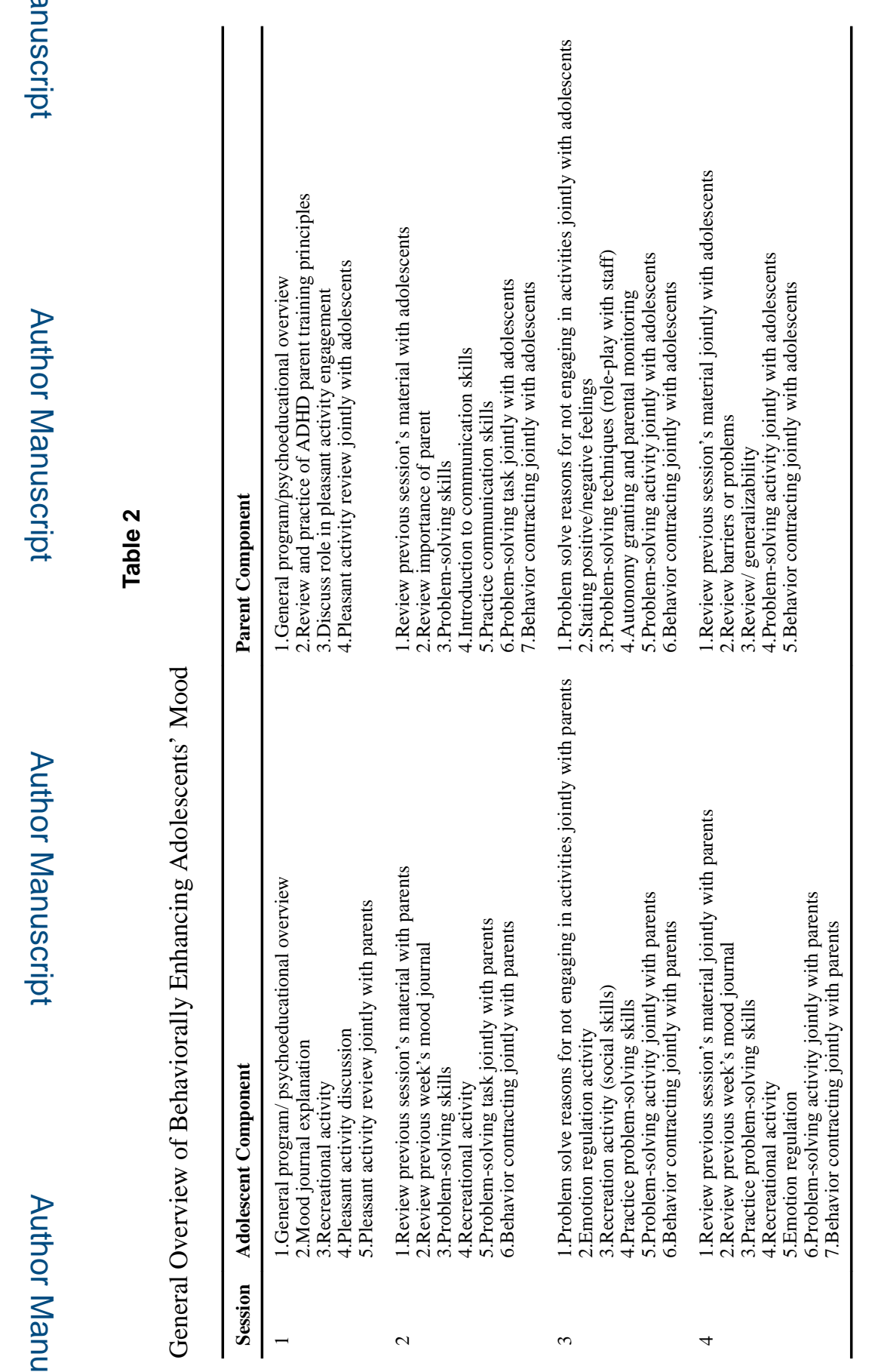

Cogn Behav Pract. Author manuscript; available in PMC 2019 November 29. 


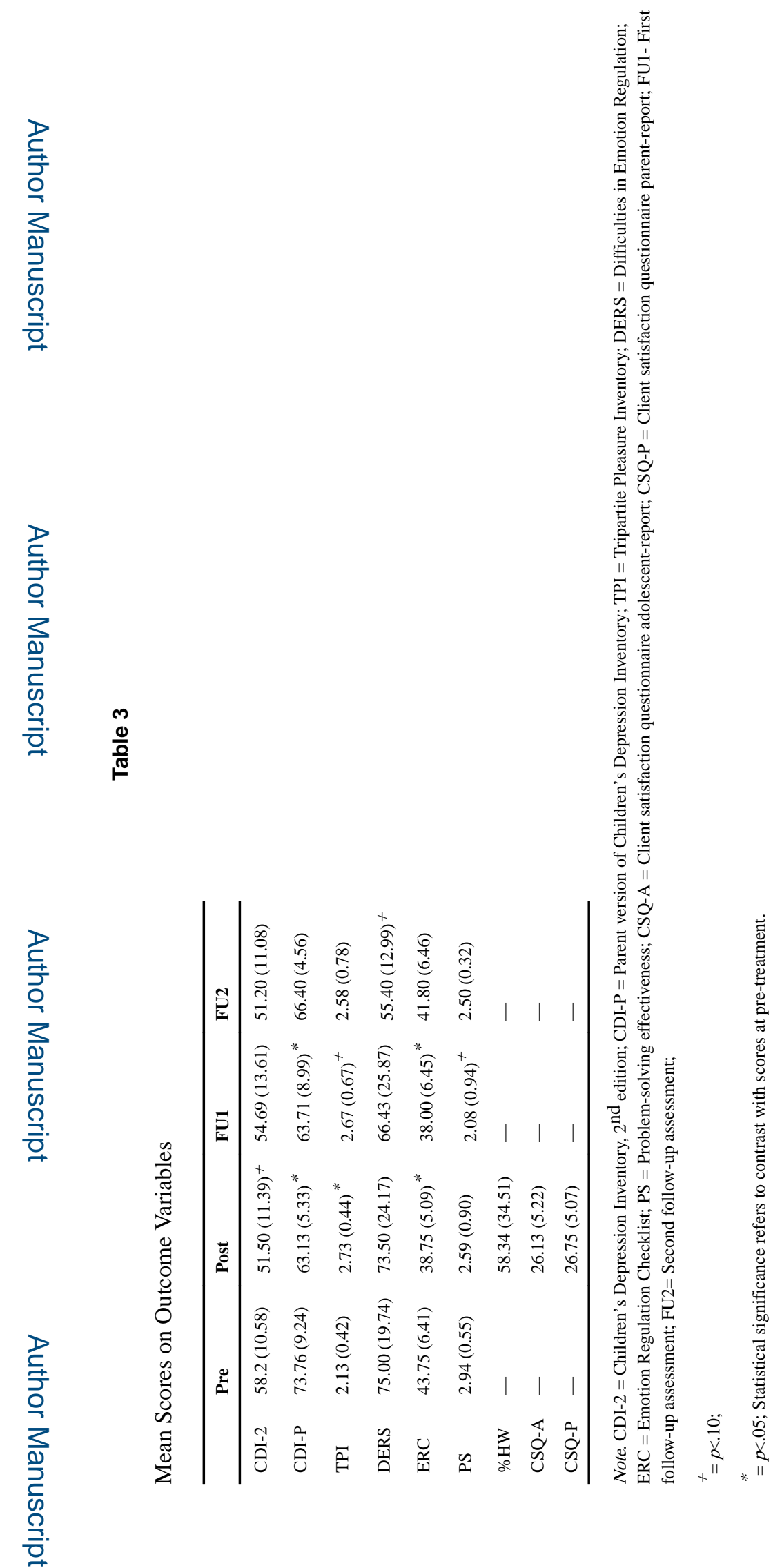

Cogn Behav Pract. Author manuscript; available in PMC 2019 November 29. 